\title{
Validation study for Large-Eddy Simulation of Forest Flow
}

\author{
George Pitchurov ${ }^{1, *}$, Christof Gromke ${ }^{2}$, Jordan A. Denev ${ }^{2}$ and Flavio Cesar Cunha \\ Galeazzo $^{3}$ \\ ${ }^{1}$ Technical University of Sofia, Bulgaria \\ ${ }^{2}$ Karlsruhe Institute of Technology, Germany \\ ${ }^{3}$ High Performance Computing Center Stuttgart (HLRS), Germany
}

\begin{abstract}
The publication presents Large-Eddy Simulation (LES) of flow over a reduced-scale wind tunnel model of a forest canopy. The final aim of the study is to determine factors responsible for damage in forests by strong winds. The wind tunnel forest was represented by an open-porous foam material for the crown layer and wooden dowels for the trunk layer. The forest model was installed in the open test section of a Goettingen-type wind tunnel and Particle Image Velocimetry (PIV) measurements were made for the acquisition of the flow field data. The numerical simulations were performed with OpenFOAM ${ }^{\circledR}$. The forest was modelled by an additional sink term in the momentum transport equations based on the leaf area density and a characteristic drag coefficient for the underlying tree specimen. Large-eddy simulations with different subgrid-scale (SGS) turbulence models were carried out and compared to wind tunnel data. The Smagorinsky SGS model outperformed the dynamic Lagrangian SGS model in the windward edge region (within a distance of approximately 2 tree heights from the leading edge) whereas the dynamic Lagrangian SGS model showed a better performance for regions farther downstream.
\end{abstract}

\section{Introduction}

Deeper understanding of the interaction between airflow and forest canopy may help to shed more light on the mechanism of forest damage by strong wind gales as well on the mass transfer of carbon dioxide and oxygen between atmospheric air and tree leaves. Increasing numbers of field and model studies of the velocity and turbulence characteristics of the flow above the forest canopy were performed in the recent years [1-4]. In particular, the number of numerical studies has increased, among which Large-Eddy Simulations (LES) gain an increasing popularity. Investigations focused on differences between various tree species, where, for simplicity, the forest was assumed to be a homogeneous continuum. The present study is motivated by the need to refine the value of the drag coefficient, resp. the momentum sink within the forest region, as well as the value of turbulent viscosity which largely determines the shape of the velocity profiles with height.

\footnotetext{
* Corresponding author: george@tu-sofia.bg
} 
In his experimental work with a wind tunnel forest model Gromke [5] identified that the streamwise mean velocity field was independent of Reynolds number for $\mathrm{Re}_{\mathrm{h}} \geq 31000$, where the length scale h equals the tree height, and that other flow quantities (fluctuation velocity, Reynolds stress, vorticity and turbulence kinetic energy) become independent for $\operatorname{Re}_{\mathrm{h}} \geq 50$ 000. The numerical study employed Large-Eddy Simulation for the Reynolds number independent range using the open source Computational Fluid Dynamics (CFD) code OpenFoam v.5. The calculations were performed on the Baden-Wurttemberg High Performance Computing (HPC) UC1 cluster in Karlsruhe using parallel computation with 4 to $16 \mathrm{CPU}$ cores. The standard Smagorinsky and the Dynamic Lagrangian subgrid-scale models were used for the computations. Two versions of the Smagorinsky subgrid-scale model were employed: with standard settings and with an increased value of the Smagorinsky constant. This was done in an attempt to address the observed discrepancy in the shape of mean velocity profiles in the forest canopy.

\section{Methods}

\subsection{Wind tunnel forest model}

The present work replicates the forest model from the wind tunnel study of Gromke [5,6], and uses the flow field data for validation. The experimental setup consists of a forest model installed in the test section of a Goettingen-type wind tunnel, Fig. 1. Upstream of the test section is a fetch section that contains spires and roughness elements to accommodate the velocity profiles as close to a fully developed flow as possible.

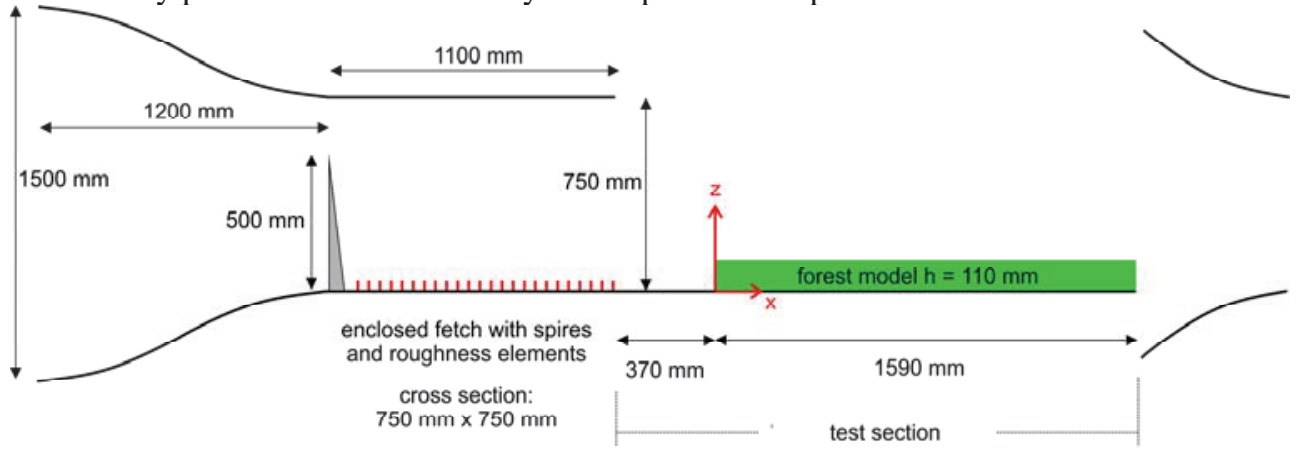

Fig. 1. Experimental setup and dimensions of the wind tunnel forest model.

The crown layer of the reduced-scale forest model $(M=1: 200)$ is composed of individual trees whose conical crowns were made using water jet cutting of a polyester foam with an isotropic pore structure. The trunk layer was represented by prefabricated cylindrical wooden dowels. This two-layer tree structure required separate treatment of the drag coefficient for each layer. Further details on the materials and methods of the physical experiment can be found in $[5,6]$.

\subsection{Computational domain}

When designing the computational domain the goal was to create an air-tight (possibly following closely the streamlines) geometry that will minimize the ambiguity in the definition of boundary conditions. In the final design, the domain enclosed the test section with the forest model, part from the fetch section and the intake of the wind tunnel (Fig. 2). The domain was extended further downstream from the wind tunnel intake to allow for 
established unidirectional flow and thus avoid recirculation at the outlet, which would introduce ambiguity in the boundary condition definition.

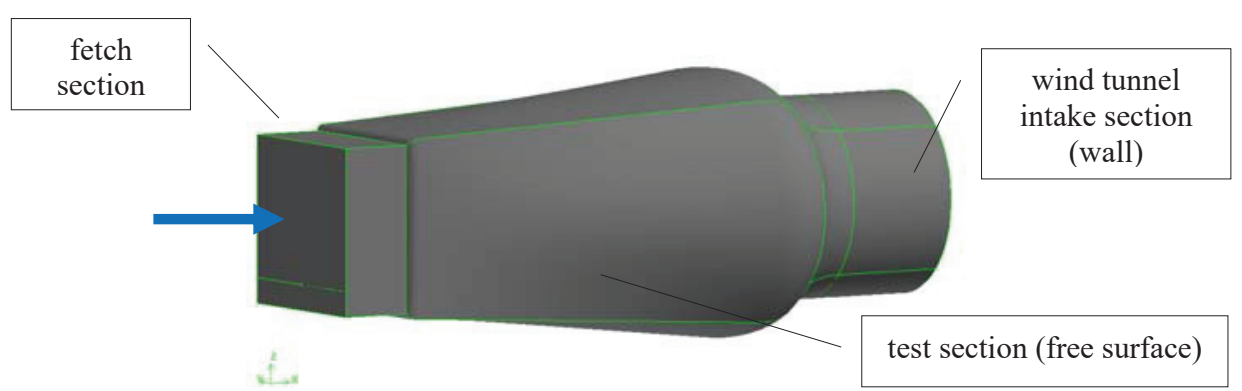

Fig. 2. Computational domain.

The air is discharged from the fetch into the open test section as a jet. This jet entrains the surrounding still air and expands. The free surface of the domain was designed in a way to capture this expansion and incorporate the jet within the computational geometry. The growth rate of the mixing layer between the jet and the surrounding air depends on the physical variable used for its definition. For example, [7] found that the growth rate of velocity thickness in a mixing layer is similar to the one of the vorticity thickness, both being higher than the growth of momentum and energy thickness. Yet, all of them have growth rates smaller than the visual thickness. Experiments $[8,9]$ identified that the growth rate of a round jet is smaller than that of a plane jet, which is on itself smaller than the growth of a mixing layer, but generally all growth rates fall in the range $0.09 \div 0.12$ (spanwise-to-streamwise ratio). Since there are several variables to consider, the free surface boundary was created to satisfy the largest growth rate.

The computational mesh is shown in Fig. 3. It consists entirely of hexahedral elements, as they can fill more economically the computational domain compared to tetrahedral or pyramidal cells of the same element size. The resulting number of elements is 2.4 million, with worst element skewness equal to 0.85 . Refinements were introduced where necessary with gradual expansion ratios to satisfy the high mesh requirements of LES models.

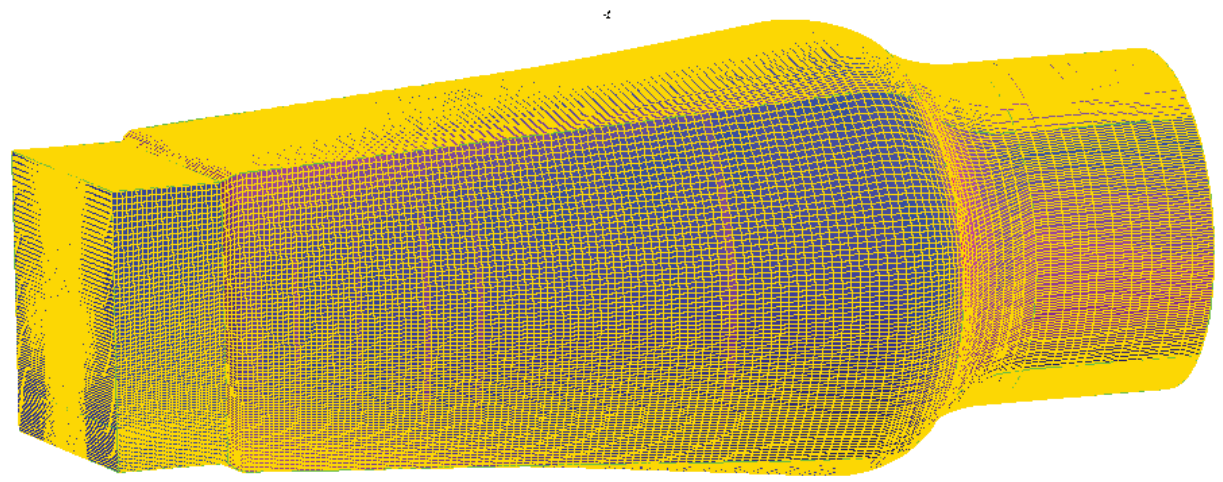

Fig. 3. Computational mesh. 


\subsection{Boundary conditions}

Tree types of boundary conditions are of particular interest: the inlet, the outlet and the free surface that should ideally coincident with the jet boundary. The computational inlet is located within the fetch section of the wind tunnel and as such exhibits only a partially developed boundary layer profile. PIV measurements were not available for the location of the domain inlet as the laser sheet could not reach that deep into the fetch (Fig. 1). Prescribing an adequate velocity profile based on a physical law proved to be a challenging task. In the following, mean velocity profiles for turbulent boundary conditions are briefly reviewed.

Boundary layer theory [10] states that the sum of viscous and turbulent shear stress is constant over height in the viscous and inertial sublayer and equals the wall shear stress:

$$
v \frac{\partial U}{\partial y}-\overline{u^{\prime} v^{\prime}}=\left.v \frac{\partial U}{\partial y}\right|_{y=0}=\tau / \rho=U_{\tau}^{2}
$$

Away from the wall, but still within the near-wall layer, the shear stress evolves entirely due to turbulent fluctuations. These fluctuations were measured with PIV and a friction velocity $U=0.47 \mathrm{~m} / \mathrm{s}$ was determined at the wall. This value was substituted in the log-law to yield the velocity profile according to Eq. (2) below:

$$
U(z)=\frac{U_{\tau}}{\kappa} \ln \left(\frac{z+z_{0}}{z_{0}}\right)
$$

The surface roughness $\mathrm{z}_{0}$ was determined from the PIV measurements of Gromke [5] by using an interpolation with the log-law relationship once the friction velocity was determined. Since measurements considered in this study were Reynolds number independent, the surface roughness emerged as a constant with a value in the range $\mathrm{z}_{0}=0.0013 \div 0.0014 \mathrm{~m}$.

Furthermore, the velocity defect law [11] and the law of the wake [12] were considered to prescribe the velocity profile at the inlet. They both depend on a polynomial wake function whose coefficients were taken from [13]. The calculations required extra coefficients like a wake parameter, smooth wall log-law intercept and velocity-defect intercept as can be found in [11]. A further attempt to represent the velocity profile by a commonly used relationship was via a power law formulation referred to by [14] used to represent in a simplistic way an atmospheric boundary layer profile:

$$
\frac{U}{U_{\tau}}=\left(\frac{z}{z_{r e f}}\right)^{\alpha}
$$

The power law exponent was set to 0.17 corresponding to a moderately rough terrain. It should be stressed that although the power law does not carry the sound theoretical basis as the equilibrium log-law or the defect law, [15] argues that this is not the case, particularly at low-Reynolds numbers. For completeness, the study also applied a power law formulation for the inlet velocity profile.

After all parametric constants were set, with some of them tuned for the case at hand, the aforementioned laws produce the profiles shown in Fig. 4. The velocity profile in the wind tunnel measured at position $\mathrm{x} / \mathrm{h}=-1$ is also included for comparison. Visual inspection shows that the shape of the measured data cannot be well replicated by any of 
the theoretical relationships. The reason is that because of the relatively short fetch section, the boundary layer is possibly not fully developed. For this purpose a curve-fit was carried out over a set of classical equations to yield a best fit in the form of an exponential relation $\mathrm{U}=\mathrm{a}+\mathrm{b} \exp (-\mathrm{z} / \mathrm{c})$, with coefficients $\mathrm{a}=8.14, \mathrm{~b}=-7.10, \mathrm{c}=0.20$. The fit improved, as can be seen in Fig. 4, although it had the drawback of producing a non-zero velocity at the wall. This necessitated the deployment of piece-wise profile later.

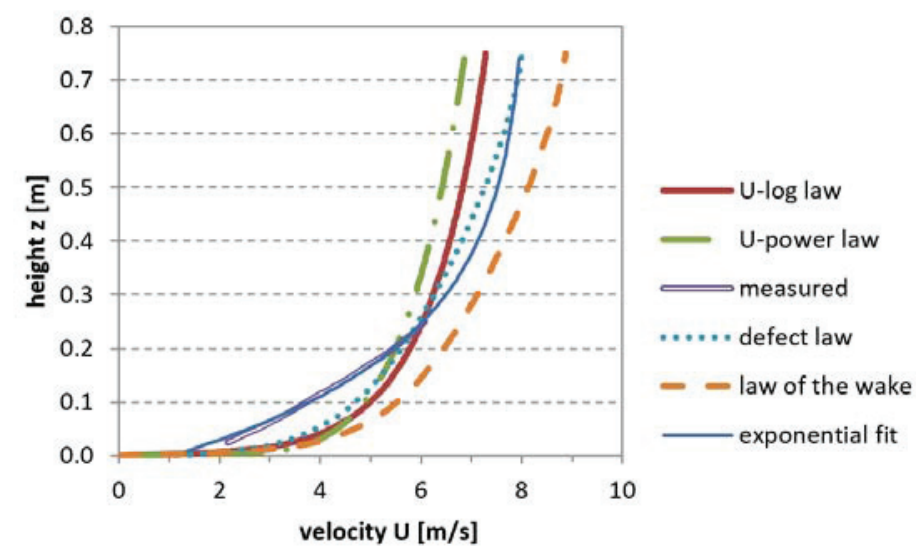

Fig. 4. Mean velocity profiles at the domain inlet and at $\mathrm{x} / \mathrm{h}=-1$ for measured data.

To maintain turbulent flow in a Large-Eddy Simulation it is necessary to supply a turbulent flow at the inlet. Once the mean velocity profile was specified it has to be randomized around the time-mean by using a stochastic algorithm. Two options existed for this purpose. One is the built-in boundary condition of OpenFOAM called 'turbulent inlet'. This condition uses a random number generator to introduce random oscillations with a user-defined intensity. These oscillations are Gaussian random, which is not a genuine characteristic of turbulence, but does allow to maintain turbulence within the domain. The other option would be a custom-made turbulence generator that creates eddies based on a user-defined length scale.

The computational outlet was placed downstream of the wind tunnel intake as a pressure outlet boundary condition. This condition requires that the pressure be specified while all other variables are assumed to have zero gradient in the normal direction. Pressure measurement data were not available at the intake, neither downstream from it, so the pressure had to be estimated. Since the wind tunnel is a closed circuit, it preserves the volume flow rate, meaning that the nozzle discharge flow rate is equal to the intake flow rate. This is only given if there is no flow through the surrounding free surface of the domain (Fig. 2). In order to achieve this, the pressure value at the wind tunnel intake was adjusted iteratively until a zero flow rate through the surrounding free surface was attained (Fig. 5).

The surrounding free surface of the domain was permeable to the flow, as is the case for the test section of the Goettingen-type wind tunnel. At this jet boundary, the pressure equals the ambient pressure ( $0 \mathrm{~Pa}$ gauge), however, the velocity of the entrained air is not known. This required that pressure and velocity were treated in a specific way, by the so-called entrainment boundary condition [16]. In this boundary condition the ambient pressure is specified while the remaining variables are assumed to be zero gradient at all times, with the exception of the tangential velocity. In case of outflow the pressure is assigned to the specified value, while for inflow it is reduced by the dynamic pressure of the inflowing fluid: 


$$
p=\left\{\begin{array}{c}
p_{0} \rightarrow \text { outflow } \\
p_{0}-\frac{1}{2}\left|U^{2}\right| \rightarrow \text { inflow }
\end{array}\right.
$$

In this way, the pressure is adjusted correctly according to the Bernoulli equation. The idea behind this treatment is that the condition is a standard treatment in case of outflow, while for inflow the normal velocity is allowed to find its own value.

\subsection{Implementation of vegetation}

In the numerical simulation the trees were represented by a height-dependent bulk source term to represent them in the momentum equations. The source terms for the trunk and crown layer were implemented analogously, however, their derivation relied on different physical principles of flow resistance. Whereas in the trunk layer the flow resistance originates from form drag at the tree trunks, the flow resistance in the crown layer is also due to surface friction at the leaf surfaces. In the implementation, the drag was taken to be height-independent in the trunk layer and height-dependent (decreasing with height) in the crown layer, hence reflecting the vertical distribution of leaf surface area. The general form of the source (more precisely sink) term in the Navier-Stokes equations follows the work of Dupont and Brunet [17] and is expressed through the form:

$$
S_{m o m, i}=-0.5 \cdot C_{d} \cdot C_{P P I-10} \cdot \sqrt{\tilde{u}_{j} \tilde{u}_{j}} \cdot \tilde{u}_{i}
$$

The swung dash denotes instantaneous LES-filtered velocity components. The coefficient $C_{d}=0.2$ is the characteristic drag resistance coefficient for the vegetation. $C_{P P I} 10$ is a resistance coefficient of the employed PPI-10 foam material. It accounts for the conical shape of the modelled trees and the volume displaced by them and is height-dependent. The resistance in the trunk layer follows the form of the above equation. Consistent with the features of the physical model used for the wind tunnel measurements, the drag coefficient in this layer is considerably lower than that in the crown layer [5,6].

\section{Results}

As a first step in the calculations the appropriate static pressure at the wind tunnel intake had to be determined and assigned to the simulations. The pressure controls the proportion between the outflow rates through the intake and through the free jet boundary. Since the wind tunnel is a closed circuit arrangement, the wind tunnel supply and intake flow rates must be equal, leaving zero net flowrate for the free surface. Thus, the correct pressure value of the intake emerges when the resulting flow rate through the free surface boundary is zero. Fig. 5 presents the time-evolution of flow rate at the free surface boundary along with its average for different values of assigned intake pressures. It was found that an intake vacuum of 5.6 Pa delivers a free-surface flow rate close to zero, thus satisfying flow continuity within the wind tunnel. 


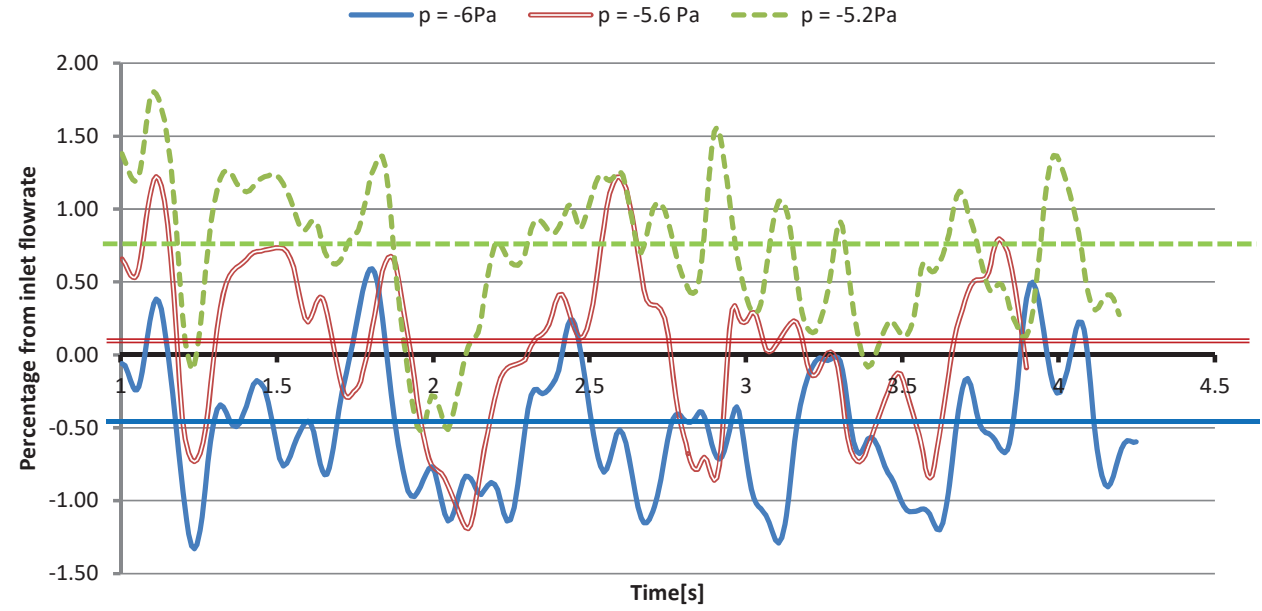

Fig. 5 Free surface flow rate as a percentage of nozzle flowrate, instantaneous and average values.

An overall impression of the flow field can be gained by examining Figs. 6 and 7. Fig. 6 shows a plot of the mean velocity vectors in the spanwise-central plane of the domain obtained with the Smagorinsky model. The presence of the forest source terms is clearly manifested by the markedly reduced velocity in the crown layer. The resistance of the forest induces a recirculation in front of its windward edge. The trunk layer exhibits smaller drag to the flow and thus a substantial share of the flowrate is directed underneath the crowns into the trunk layer. The trunk drag, however, accumulates downstream and finally the velocity is reduced even within the trunk layer with the airflow diverging mostly sideways.

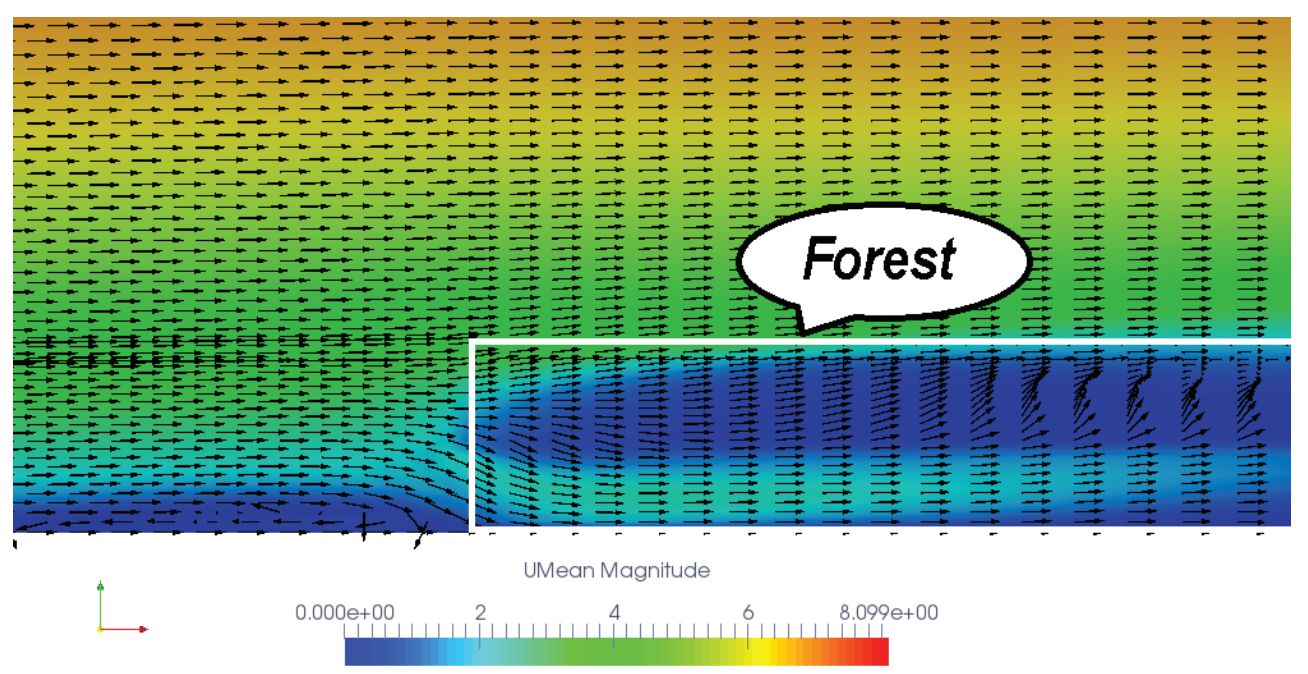

Fig. 6 Mean velocity vectors $[\mathrm{m} / \mathrm{s}]$ in the spanwise-central plane.

Fig. 7 shows a snapshot of the instantaneous velocity magnitude in the same plane calculated also with the Smagorinsky subgrid-scale model. The color contours indicate the shear layer above the forest canopy where extra vortex generation is expected to occur. The boundary layer develops more quickly and distinctly due to the drag from trees compared to the fetch region. 

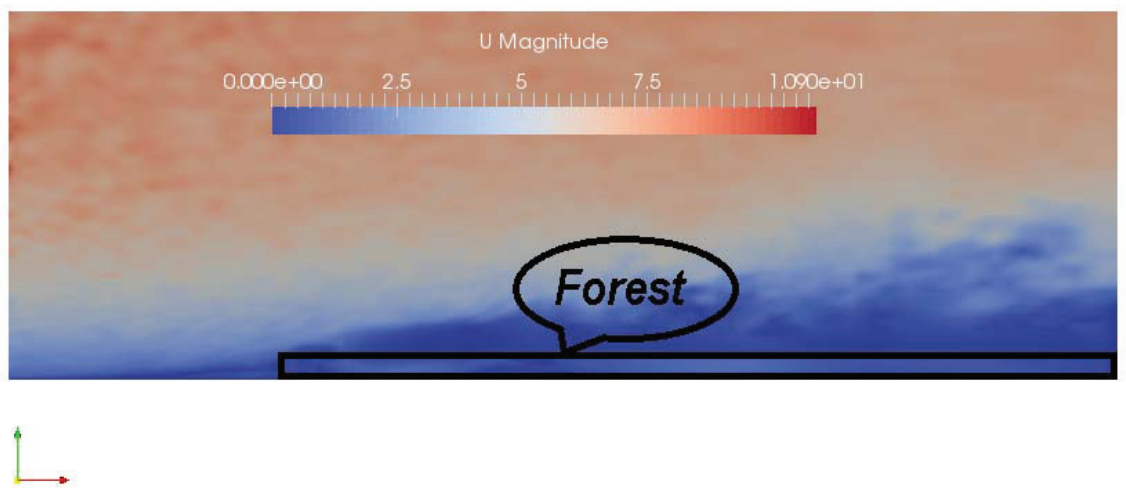

Fig. 7 Contours of instantaneous velocity $[\mathrm{m} / \mathrm{s}]$ in the spanwise-central plane.

To validate the results, comparison of simulations and experiments along vertical mean velocity profiles was performed. PIV measurements of the velocity were evaluated along vertical lines arranged equidistantly within the spanwise-central plane. Starting upstream of the forest leading edge, the sampling lines were spaced at distance $0.5 \mathrm{~h}$ from each other, where $\mathrm{h}$ is the tree height. A selection of mean velocity profiles over these lines is presented in Fig. 8 for three versions of SGS-models, all obtained with the exponential curve from Fig. 4 as mean velocity inlet condition.

The three versions of SGS-models presented in Fig. 8 consist of the standard Smagorinsky model, the "High Smagorinsky Constant"-model with four times increased value of the model constant, and the dynamic Lagrangian model as implemented in OpenFOAM described originally in [18]. The latter model proved to be numerically less stable and the Courant-Friedrichs-Lewy (CFL) condition was decreased from 0.5 to 0.4 . The reason to introduce the "High Smagorinsky Constant"-model was to check how sensitive are the results with respect to the increase of this constant and to assess how this increase influences the sink term within the canopy shown in equation (5).

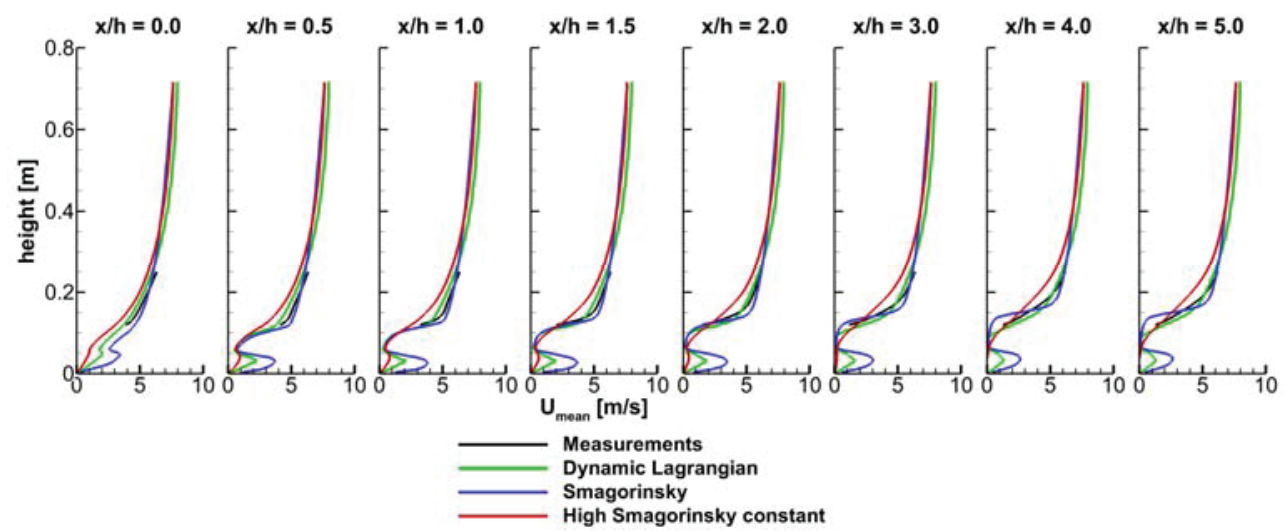

Fig. 8 Mean velocity profiles compared with PIV measurements at different streamwise coordinates $\mathrm{x} / \mathrm{h}$.

From Fig. 8 it can be seen that in the above canopy region the knee in the profiles of the PIV measurements in the range $\mathrm{x} / \mathrm{h} \leq 1.5$ is most exactly reproduced by the Smagorinsky model. In this region the Smagorinsky model exhibits the closest agreement 
with the measurements. Starting with $\mathrm{x} / \mathrm{h} \geq 2.0$, the results from the dynamic Lagrangian SGS model agree better with the experiment. In this region, the vertical shape of the profile for the Smagorinsky model develops slower and therefore starts to deviate more and more from the measurements. The High Smagorinsky constant SGS model, due to its diffusive nature, shows a too fast boundary-layer development above the trees and therefore it deviates the most from the experimental data.

As it is difficult to figure out a clear superiority of either the standard Smagorinsky model or the dynamic Lagrangian model only by visual inspection of Fig. 8, the two SGS models are compared in terms of an error assessment indicator: the Mean Bias Error (MBE) which is the sum of the observed (measured) minus predicted (simulated) values normalized by the number of samples. Results are presented in Table 1. Whereas the Smagorinsky SGS model shows an overprediction in the windward leading edge region up to $\mathrm{x} / \mathrm{h}=2$ and an underprediction farther downstream, the dynamic Lagrangian SGS model shows the opposite behavior with overpredictions up to $\mathrm{x} / \mathrm{h}=1.5$.

Table 1. Mean Bias Error (MBE) at various vertical profiles $\mathrm{x} / \mathrm{h}$, see Fig. 8

\begin{tabular}{|c|c|c|c|c|c|c|c|c|c|}
\hline $\mathbf{x} / \mathbf{h}$ & SGS-model & $\mathbf{0 . 0}$ & $\mathbf{0 . 5}$ & $\mathbf{1 . 0}$ & $\mathbf{1 . 5}$ & $\mathbf{2 . 0}$ & $\mathbf{3 . 0}$ & $\mathbf{4 . 0}$ & $\mathbf{5 . 0}$ \\
\hline \multirow{2}{*}{$\mathrm{MBE}$} & Smagorinsky & -0.15 & -0.14 & -0.23 & -0.29 & -0.38 & 0.06 & 0.16 & 0.10 \\
\cline { 2 - 9 } & dyn. Lagrangian & 0.35 & 0.42 & 0.34 & 0.20 & -0.02 & 0.08 & -0.03 & -0.28 \\
\hline
\end{tabular}

Fig. 8 helps to compare the models in the trunk layer $(\mathrm{z}<0.055 \mathrm{~m})$. In this region the porosity is less than in the crown region. In the vicinity of the ground, the High Smagorinsky constant model quickly damps the velocity so that downstream, at $\mathrm{x} / \mathrm{h} \geq 3$, the model predicts practically no movement of the fluid between the trunks. This is substantially different from the results with the classical Smagorinsky model, where the flow in the trunk layer still reaches almost $3 \mathrm{~m} / \mathrm{s}$ at a distance of $\mathrm{x} / \mathrm{h}=5.0$. The dynamic Lagrangian model approaches the forest edge $(\mathrm{x} / \mathrm{h}=0)$ with an already retarded speed in the trunk region and its velocity for all shown profiles is approximately half of the velocity of the Smagorinsky model.

The High Smagorinsky constant model is close to the classical Smagorinsky model in the crown layer $(0.055<\mathrm{z}<0.110 \mathrm{~m})$ at $\mathrm{x} / \mathrm{h}=1$, however due to increased diffusivity, further downstream it exhibits higher velocities in the upper parts of the crowns than the other two models. This leads to the conclusion that the effects of the turbulent viscosity from all SGS models are not completely dominated by the action of the porous terms introduced by equation (5) and hence the SGS models are also active within the complete layer of the canopy.

Computations of turbulent Reynolds stresses showed some discrepancies with the PIV measurements. In an effort to resolve this issue another synthetic turbulence generator was applied at the domain inlet. This generator introduces fluctuations that retain most of the characteristics of turbulent flows. The generator deployed was based on the digital filter proposed by Klein et. al. [19]. The turbulence length scale was assigned equal to $0.05 \mathrm{~m}$ in all dimensions and turbulence intensity set to $10 \%$ of the inlet velocity, which roughly corresponded to PIV measurements in the $\mathrm{x} / \mathrm{h}=-1$ section. Fig. 9 shows preliminary results of the instantaneous velocity in the spanwise-central plane using the synthetic generator. Inspection of the figure shows that the domain free surface satisfactorily coincides with the visual jet boundary, which was one of the goals when designing the computational geometry. It also illustrates the presence of flow inside the trunk layer downstream of the forest windward edge. 


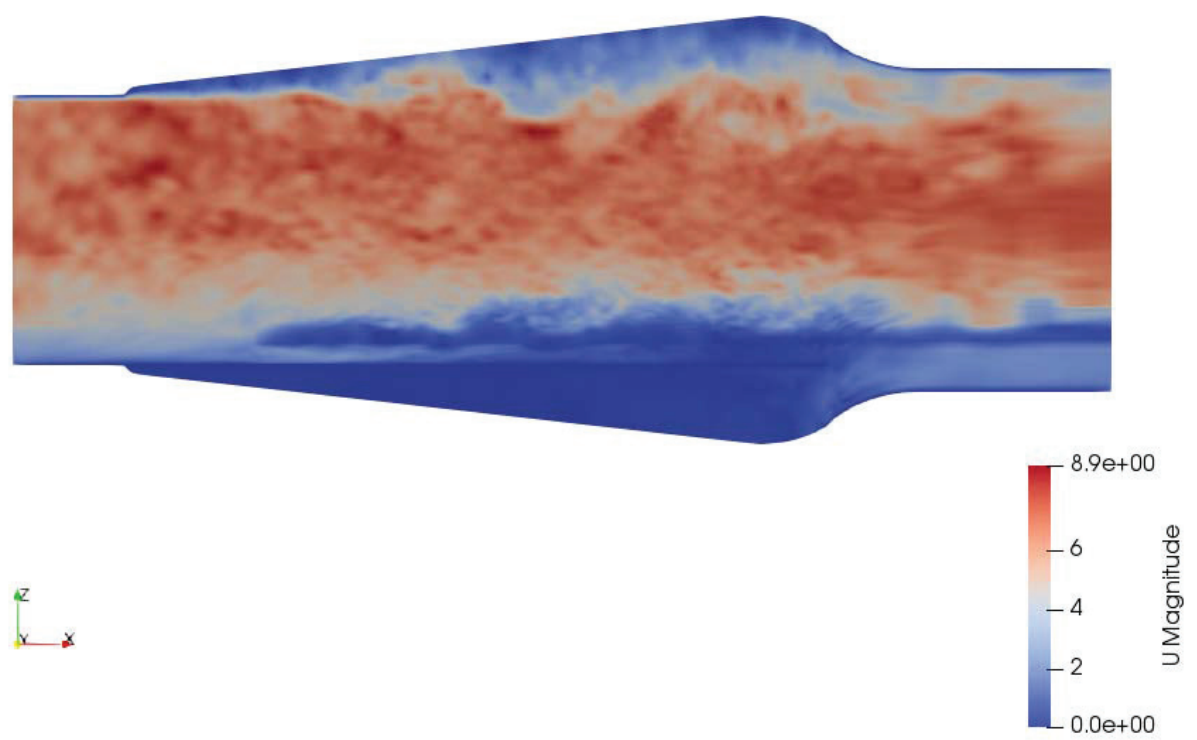

Fig. 9 Instantaneous velocity $[\mathrm{m} / \mathrm{s}]$ in the spanwise-central cross section of the domain.

\section{Conclusions}

A large-eddy simulation of the flow in a two-layer forest model was performed and compared to wind tunnel PIV measurements. A proper domain shape and boundary conditions ensured that the problem was numerically well setup. The forest drag was represented by a bulk sink term in the momentum equations. Analysis of mean velocity profiles showed that airflow penetrates deeply in the trunk layer because of its relatively small resistance to the flow. However, the leaves and branches in the crown layer introduce significant drag. Observations inside the forest are only possible with the help of a numerical simulation, since PIV measurements do not allow to measure inside the forest canopy. Therefore, mean velocity validation was done only for the flow above the canopy.

The validation used the exponential fit shown in Fig. 4 for the mean velocity profile superimposed by random oscillations for the inflow turbulence. Three variants of subgridscale (SGS) models were tested. The Smagorinsky model showed slightly better results in the upstream part and at the leading edge of the forest (up to $\mathrm{x} / \mathrm{h} \leq 1.5$ ) while the dynamic Lagrangian model performed better farther downstream. The third model - a variant of the Smagorinsky model with a four times higher constant proved to be too diffusive, however, its interaction with the flow in the tree crowns indicated that the influence of the SGS models is not negligible even in the region of high flow resistance.

The validations revealed discrepancies that required a further revision of the inlet boundary condition to address the absence of a fully developed boundary layer approach profile. In an effort to resolve this discrepancy, another inflow turbulence generator was employed and first results show reasonable behavior and will be presented in more detail in future together with an analysis of second order flow characteristics.

The numerical simulations of this work were carried out on the supercomputer bwUniCluster at Steinbuch Centre for Computing at the Karlsruhe Institute of Technology. 


\section{References}

1. K. Bai, J. Katz, C. Meneveau, Turbulent flow structure inside a canopy with complex multi-scale elements. Bound.-Layer Meteorol. 155, 435-457 (2015)

2. B. Conan, S. Aubrun, B. Coudour, K. Chetehouna, J.P Garo, Contribution of coherent structures to momentum and concentration fluxes over a flat vegetation canopy modelled in a wind tunnel. Atmos. Environ. 107, 329-341 (2015)

3. B. Ruck, C. Frank, M. Tischmacher, On the influence of windward edge structure and stand density on the flow characteristics at forest edges. Eur. J. For. Res. 131, 177-189 (2012).

4. S. Aubrun, R. Koppmann, B. Leitl, M. M€ollmann-Coers, A. Schaub, Physical modelling of a complex forest area in a wind tunnel-comparison with field data. Agric. For. Meteorol. 129, 121-135 (2005)

5. C. Gromke, Wind tunnel model of the forest and its Reynolds number sensitivity, Journal of Wind Engineering and Industrial Aerodynamics 175 53-64 (2018).

6. C. Gromke, B. Ruck, On Wind Forces in the Forest Edge Region during Extreme Gust Passages and Their Implications for Damage Patterns. Boundary-Layer Meteorology, Vol. 168, pp. 269-288 (2018)

7. D. Yoder, J. DeBonis, N. Georgiadis, Modeling of turbulent free shear flows, NASA/TM-2013-218072, AIAA Paper 2013-2721, E-18747, https://ntrs.nasa.gov/archive/nasa/casi.ntrs.nasa.gov/20140002507.pdf (2013)

8. L. J. S. Bradbury, "The Structure of a Self-Preserving Turbulent Plane Jet," Journal of Fluid Mechanics, Vol. 23, No. 1, pp. 31-64 (1965).

9. I. Wygnanski, H.E. Fiedler, "Some Measurements in the Self-Preserving Jet," Journal of Fluid Mechanics, Vol. 38, No. 3, pp. 577-612 (1969)

10. J. Picket, Turbulent Flows: Models and Physics, Springer, ISBN-10: 3540654119 (1999)

11. J.S. Connelly, M.P. Schultz, K.A. Flack, Velocity-defect scaling for turbulent boundary layers with a range of relative roughness, Experiments in Fluids 40: 188-195 DOI 10.1007/s00348-005-0049-x (2006)

12. D. Coles, The law of the wake in the turbulent boundary layer. Journal of Fluid Mechanics, 1(2), 191-226. doi:10.1017/S0022112056000135 (1956)

13. P.S. Granville, A modified law of the wake for turbulent shear layers. J Fluids Eng 98:578-580 (1976)

14. VDI 3783. Environmental meteorology, physical modelling of flow and dispersion processes in the atmospheric boundary layer. Verein Deutscher Ingenieure (2000)

15. K.R. Sreenivasan, "The Turbulent Boundary Layer," Frontiers in Experimental Fluid Mechanics, edited by M. Gad-el-Hak, Springer-Verlag,Berlin, pp. 159-209 (1989)

16. OpenFoam user guide, https://cfd.direct/openfoam/user-guide/v5-boundaries

17. S. Dupont, Y. Brunet, Coherent structures in canopy edge flow: a large-eddy simulation study. J Fluid Mech 630:93-128, doi:10.1017/S0022112009006739 (2009)

18. C. Meneveau, T.S. Lund, W.H. Cabot, A Lagrangian dynamic subgrid-scale model of turbulence. Journal of Fluid Mechanics, 319, 353-385 (1996)

19. M. Klein, A. Sadiki, J. Janicka - A digital filter based generation of inflow data for spatially developing direct numerical or large eddy simulations - Journal of Computational Physics, Volume 186, Issue 2, pp 652-665 https://doi.org/10.1016/S0021-9991(03)00090-1 (2003) 